\title{
L'APPLICATION DU CALCUL DES PROBABILITÉS AUX PROBLÈMES D'EXPLOITATION DES RÉSERVOIRS
}

\section{INTRODUCTION AUX PROCESSUS DE MARKOFF}

\author{
PAR \\ J. BERNIER *
}

Cette courte présentation est une préface aux articles de MM. Roux : «Relations entre la capacité d'un réservoir et son usage - Applications aux oueds d'Algérie », et Bernier : «La théorie stochastique des réservoirs », qui font appel aux propriétés fondamentales des processus markoviens.

En dehors même de l'intérêt qui s'attache à un exposé plus détaillé de notions introduites, trop rapidement peut-être, dans les deux études citées, cette présentation est justifiée par l'importance du rôle joué par les processus markoviens dans des domaines aussi divers que : la physique nucléaire, l'électronique, la chimie, l'astrophysique, la biologie, la démographie, l'économie, la recherche opérationnelle, etc.

\section{Description}

Le terme processus de Markoff désigne un modèle mathématique propre à décrire l'évolution dans le temps de systèmes aléatoires particuliers.

Considérons un processus aléatoire $X(t)$ fonction du temps, c'est-à-dire une grandeur $X$ pour laquelle sont donnés, d'une part, un domaine (ensemble de tous les états ou valeurs possibles de $\mathrm{X}$ ) et, d'autre part, une loi de probabilité qui, à chaque état, fait correspondre la probabilité que $\mathrm{X}$ soit en cet état à l'instant $t$.

On connaît les modèles de probabilité basés sur l'hypothèse d'indépendance que, en supposant une

\footnotetext{
* Tugénieur au Centre de Recherches et d'Essais de Chatou (E.D.F.)
}

époque quelconque considérée comme l'instant présent, on peut transcrire ainsi : la loi de probabilité des états futurs du système, connaissant les états passés et présent (loi conditionnelle) est indépendante de ces états passés et présent.

On sait que de nombreux phénomènes naturels physiques ou autres sont en bon accord avec cette hypothèse.

Une restriction toute naturelle de cette hypothèse d'indépendance est la suivante :

La loi de probabilité conditionnelle des états futurs du système, connaissant les états passés et présent, est indépendante des états passés et ne dépend que de l'état présent.

Que cette hypothèse, dite markovienne, puisse ètre réaliste dans de nombreuses circonstances très variées, les exemples suivants, choisis parmi beaucoup d'autres, le montreront.

\section{Exemples}

Biologie. GÉnétigue. DÉmographe (les processus DE RENOUVELLEMENTS).

Considérons une population quelconque, humaine ou bactérienne, etc. Les différents états $\mathrm{E}_{n} \mathrm{du}$ processus sont définis par les différents effectifs $n$ possibles pour cette population. Les transitions possibles sont $\mathrm{E}_{n} \rightarrow \mathrm{E}_{n+1}$ ou $\mathrm{E}_{n} \rightarrow \mathrm{E}_{n-1}$ ou $\mathrm{E}_{n} \rightarrow \mathrm{E}_{n}$. Si donc le processus est dans l'état $\mathrm{E}_{n}$ à l'instant $t$ (effectif $n$ ) il passera, à l'instant $t+\Delta t$, soit dans l'état $\mathrm{E}_{n+1}$ avec une probabilité $\lambda_{n} \Delta t$ (naissance 
d'un individu), soit dans l'état $\mathrm{E}_{n-1}$ avec une probabilité $\mu_{n} \Delta t$ (mort d'un individu) soit dans l'état $E_{n}$ avec une probabilité $1-\left(\lambda_{n}+\mu_{n}\right) \Delta t$ (statu qulo).

\section{Physique nucléalre (réacteurs nuclétires).}

L'exemple présent concerne le problème de la variation d'énergie de neutrons évoluant dans un milieu (modérateur) constitué de noyaux atomiques de différentes sortes. Les variations d'énergie surviennent au moment de la collision d'un neutron avec un noyau d'un type donné. La probabilité de cette collision dépend de l'énergie du neutron à cet instant; s'il y a collision, la probabilité qu'une variation d'énergie se produise, d'une part, et la probabilité de l'intensité de cetle variation, de l'autre, dépendent seulement de l'énergie du neutron au moment de la collision. Nous sommes done ici dans le cadre de l'hypothèse markovienne.

\section{RECHERCHE OPÉRATIONNELLE.}

Le contenu d'un réservoir $\mathrm{X}(t)$ à un instant $t$ est un processus aléatoire dont l'évolution est réglée, d'un côté, par le débit de sortie défini par une règle de gestion liée au contenu du réservoir et, de l'autre côté, par le débit aléatoire entrant $\mathrm{Q}(t)$. Si les $\mathrm{Q}(t)$ successifs sont indépendants en probabilité, le contenu du réservoir $\mathrm{X}(t)$ est un processus markovien. Si les $Q(t)$ sont eux-mêmes markoviens, ce n'est plus $\mathrm{X}(t)$, mais le couple $[\mathrm{X}(t), \mathrm{Q}(t)]$ qui est markovien.

\section{Historique et bibliographie}

L'hypothèse markovienne fut introduite par Markoff en 1906 comme une généralisation de l'hypothèse d'indépendance en vue d'essayer d'étendre le domaine d'application des théorèmes asymptotiques classiques du calcul des probabilités comme la loi des grands nombres, par exemple.

On trouvera des exposés très complets de la théorie dans de nombreux ouvrages dont nous citerons :

- M. Fréchet. - Recherche théorique moderne sur le calcul des probabilités; second livre : Méthode de fonctions arbitraires. Théorie des événements en chaîne dans le cas d'un nombre fini d'états possibles. Gauthier-Villars (1938);

- Blanc-lapierre el Fortet. - Théorie des fonctions aléatoires. Masson (1953);

-W. Feller. - An introduction to probability theory and its applications. Wiley and Son, New York (1957);

- Bharucka Rem. - Elements of the theory of Markoff processes and their applications. Mac Graw Hill, New York (1960).

\section{Les probabilités de transition}

Pour la simplicité, nous ne traiterons ici que le cas d'un processus ne pouvant prendre qu'une infinité dénombrable d'états possibles : $\mathrm{E}_{1}, \mathrm{E}_{2} \ldots \mathrm{E}_{k}$ ..., et observé sur une suite d'instants discrets : $t_{0}, t_{1}, \ldots t_{n} \ldots$
L'évolution aléatoire du processus est caractérisée par :

- les probabilités initiales $\mathrm{P}_{i}^{\circ}$ des états $\mathrm{E}_{i}$ à l'instant initial $t_{0}$;

- les probabilités de transition $\mathrm{P}_{i j}$ d'un état $\mathrm{E}_{i}$ à l'instant $t_{n}$, à l'état $\mathrm{E}_{\mathrm{j}}$ à l'instant $t_{n+1}$. On peut arranger les $\mathrm{P}_{i j}$ sous forme de matrice $\mathrm{P}$.

$t_{n} \begin{array}{ccccc}\mathrm{E}_{1} & \mathrm{E}_{22} & \ldots & \mathrm{E}_{k} & \ldots \\ \mathrm{E}_{1} & \mathrm{P}_{11} & \mathrm{P}_{12} & & \mathrm{P}_{1 k} \\ \mathrm{E}_{2} & \mathrm{P}_{21} & \mathrm{P}_{22} & & \mathrm{P}_{2 k} \\ \ldots & & & & \\ \mathrm{E}_{k i} & \mathrm{P}_{k 1} & \mathrm{P}_{k: 2} & \ldots & \mathrm{P}_{k i k} \\ \ldots & \ldots & \ldots & & \ldots\end{array}$

Il est clair que les sommes en lignes donnent : $\sum \mathrm{P}_{i j}=1$. Cette propriété caractérise une matrice dite stochastique.

Dans le cas général, les $\mathrm{P}_{i j}$ peuvent être fonction de $n$. Nous traiterons ici le cas homogène de $\mathrm{P}_{i j}$ indépendant de $n$.

Une transition de $\mathrm{E}_{j}$ à $\mathrm{E}_{t_{i}}$ en $n$ instants $t_{m}, t_{r+1} \ldots$ $t_{r+n}$ peut survenir à la suite de différentes séquences du type:

$$
\mathrm{E}_{j} \rightarrow \mathrm{E}_{j_{1}} \rightarrow \mathrm{E}_{j, \mathrm{i}} \rightarrow \ldots \mathrm{E}_{j_{n-1}} \rightarrow \mathrm{E}_{k i}
$$

où $j_{1}, j_{2}, \ldots \dot{j}_{n-1}$ est une séquence de $n-1$ indices quelconques d'états. Ia probabilité d'une telle transition s'écrit : $\mathrm{P}_{j j_{1}}, \mathrm{P}_{j_{1} j_{2}} \ldots \mathrm{P}_{j_{(n-1) k}}$; la probabilité $\mathrm{P}_{j k}^{(n)}$ de transition de $\mathrm{E}_{j}$ à $\mathrm{E}_{k}$ exactement en $n$ étapes s'obtient en faisant la somme de tous les produits de ce genre. Il en résulte que $\mathrm{P}_{j k}^{(n)}$ obéit à la formule de récurrence suivante:

$$
\mathrm{P}_{j l i}^{(n+1)}=\sum_{p} \mathrm{P}_{j} \mathrm{P}_{{ }^{\prime} k}^{(n)}
$$

ou plus généralement :

$$
\mathrm{P}_{j k}^{(n+m)}=\sum_{\nu} \mathrm{P}_{j{ }^{\prime}}^{(n)} \mathrm{P}_{p l i}^{(m)}
$$

11 est possible de considérer les $\mathrm{P}_{j k}^{(n)}$ comme les éléments d'une matrice $\mathrm{P}^{(n)}$. On reconnaît alors dans la relation (1) la règle de détermination des coefficients d'un produit matriciel, de telle sorte que l'on peut écrire symboliquement :

$$
\mathrm{P}(m+n)=\mathrm{P}(m) \cdot \mathrm{P}(n)
$$

L'application répétée de cette formule montre que $\mathrm{P}^{(n)}$ peut être interprétée comme la $n^{\mathrm{e}}$ puissance de la matrice $P$. On ne peut donc craindre d'ambiguïté en supprimant les parenthèses et en interprétant $m$ et $n$ comme des puissances ordinaires.

$$
\mathrm{P}^{m+n}=\mathrm{P}^{m} \cdot \mathrm{P}^{n}
$$

Cette relation est caractéristique des processus de Markoff.

Il est clair qu'un état $\mathrm{E}_{k}$ peut être atteint en partant de $\mathrm{E}_{j}$ s'il existe un $n$ tel que $: \mathrm{P}_{j k}^{(n)}>0$. Si tel est le cas de tous les états, le processus est dit irréductible. 


\section{Les probabilités absolues}

Les formules précédentes donnent les probabilités de transition d'un état dans un autre. Mais il importe également de connaître la probabilité (non conditionnelle) $\mathrm{P}_{k}^{(n)}$ d'un état $\mathrm{E}_{k}$ à un instant $t_{n}$ quelconque; on a :

$$
\mathrm{P}_{k}^{(n)}=\sum_{j} \mathrm{P}_{j}^{0} \mathrm{P}_{j k}^{(n)}
$$

Le processus homogène est dit stationnaire si cette probabilité $\mathrm{P}_{k}^{(n)}$ est indépendante de $n$. Un des problèmes importants est l'étude de l'influence de l'état initial sur la probabilité des états à l'époque $t_{n}$. On peut penser que cette influence diminue lorsque $n$ augmente, de telle sorte qu'il y a indépendance complète à la limite. Cette propriété, valable sous certaines conditions, est un des aspects de l'ergodisme que nous discuterons plus loin.

\section{Probabilités de retour}

Considérons un instant initial où le système est dans l'état $\mathrm{E}_{j}$; l'intervalle de temps $n$ qui s'écoule jusqu'au premier retour en $\mathrm{E}_{j}$ est une variable aléatoire dont la probabilité $\pi_{j j}^{(n)}$ s'écrit :

$$
\pi_{j j}^{(n)}=\mathrm{P}_{j j}^{(n)}-\sum_{r=1}^{n-1} \pi_{j i}^{(n-\nu)} \mathrm{P}_{j j}^{(p)}
$$

formule qui résulte simplement de ce que la probabilité du premier retour à $\mathrm{E}_{j}$ à l'époque $t_{n}$ est égale à la probabilité d'un retour à $t_{n}$ moins la probabilité que le premier retour ait lieu à l'un quelconque des instants $t_{1}, t_{2} \ldots t_{n-1}$ et qu'il soit suivi par un retour ultérieur à $t_{u}$.

La quantité :

$$
\pi_{j}=\sum_{n=1}^{\infty} \pi_{j j}^{(n)}
$$

représente la probabilité que, partant de $\mathbf{E}_{j}$, le système retourne ultérieurement toujours à $\mathrm{E}_{j}$.

Si :

$$
\pi_{j}=1,
$$

l'état $\mathrm{E}_{j}$ est alors persistant. Dans ce cas, il peut être caractérisé par son temps moyen de retour $\tau_{j}$ :

$$
\tau_{j}=\sum_{n=1}^{\infty} n \pi_{j j}^{(n)}
$$

La formule (3) peut être généralisée au cas où, partant de $\mathrm{E}_{i}$, le système revient à $\mathbf{E}_{j}$ pour la première fois à $t_{n}$. La probabilité correspondante s'écrit :

$$
\pi_{i j}^{(n)}=\mathrm{P}_{i j}^{(n)}-\sum_{\nu=1}^{n-1} \pi_{i j}^{(n-v)} \mathrm{P}_{j j}^{(p)}
$$

\section{Classement des états}

Les probabilités $\pi_{j}$ et $\mathrm{P}_{j j}^{(n)}$ permettent une classification des états, du plus grand intérêt pour la description de l'évolution aléatoire du système.

On a déjà vu que si $\pi_{j}=1, \mathrm{E}_{j}$ est persistant, de telle sorte que, si le système part de $\mathrm{E}_{j}$, on a la quasi-certitude qu'il y reviendra ultérieurement. Si $\pi_{j}<1, \mathrm{E}_{j}$ est dit transitoire.

Un état persistant est un état nul si le temps moyen de retour $\tau_{j}$ est infini. Il peut arriver que le temps de retour de certains états ne puisse être inférieur à une certaine limite. C'est le cas notamment de certains niveaux d'un réservoir dans lequel la loi des apports est périodique. La traduction mathématique de cette propriété est la suivante :

- un état $\mathrm{E}_{j}$ est dit périodique de période $\tau$ si $\mathrm{P}_{j j}^{(n)}=0$ pour tout $n$ qui n'est pas divisible par $\tau$ ( $\tau$ étant le plus petit entier possédant cette propriété).

Un état $\mathrm{E}_{j}$ persistant qui n'est ni nul ni périodique est dit ergodique. C'est le cas de l'ensemble des niveaux d'un réservoir pour lequel la loi des apports est stationnaire.

\section{Propriétés ergodiques d'un processus markovien}

L'ergodisme désigne un ensemble de propriétés asymptotiques ( $n$ grand) concernant des processus de Markof' irréductibles dont les états sont tous ergodiques. L'importance pratique de ces propriétés est liée au fait que, dans nombre de problèmes physiques, il importe d'étudier l'évolution du système dans une situation stable, lorsque l'influence des conditions initiales s'est atténuée au point de disparaitre. Une telle situation peut-elle exister? Sous quelles conditions ? Comment la caractériser ? C'est à ces diverses questions que l'étude de l'ergodisme permet de répondre. En traduisant verbalement les résultats mathématiques énoncés plus loin, on peut dire que si le processus markovien est irréductible et constitué uniquement d'états ergodiques, alors :

- une situation stable (stationnaire) unique est atteinte asymptotiquement; les probabilités stationnaires s'obtiennent directement à partir de la matrice de transition;

- ces probabilités sont égales aux inverses des temps moyens de retour.

Mathématiquement, les résultats suivants résultent de l'hypothèse d'états ergodiques :

$$
\lim _{n \rightarrow \infty} \mathrm{P}_{j k}^{(n)}=x_{k}=\frac{1}{\tau_{k}}
$$

Dans ce cas la distribution des $x_{k}$ est la seule stationnaire et elle vérifie :

$$
x_{k}=\sum_{j} x_{j} \mathrm{P}_{j k}
$$

Une généralisation de ces propriétés, également valable pour les processus ergodiques, est relative au comportement asymptotique des moyennes «temporelles»des $\mathrm{P}_{i j}$ (sommes de Cesaro):

$$
\lim _{n \rightarrow \infty} \frac{1}{n} \sum_{p=1}^{(n)} \mathrm{P}_{i j}^{(n)}=x_{i j} \text { finie }
$$

L'existence de ces limites a une importance pratique très grande, car elle justifie en particulier l'usage qui est fait des moyennes temporelles calculées sur une réalisation particulière du processus. 